Original Research Paper

\title{
Anthropomorphic Solid Structures n-R Kinematics
}

\author{
${ }^{1}$ Relly Victoria V. Petrescu, ${ }^{2}$ Raffaella Aversa, \\ ${ }^{3}$ Bilal Akash, ${ }^{4}$ Ronald B. Bucinell, ${ }^{5}$ Juan M. Corchado, \\ ${ }^{2}$ Antonio Apicella and ${ }^{1}$ Florian Ion T. Petrescu \\ ${ }^{1}$ ARoTMM-IFToMM, Bucharest Polytechnic University, Bucharest, (CE) Romania \\ ${ }^{2}$ Advanced Material Lab, Department of Architecture and Industrial Design, \\ Second University of Naples, 81031 Aversa (CE) Italy \\ ${ }^{3}$ Dean of School of Graduate Studies and Research, American University of Ras Al Khaimah, UAE \\ ${ }^{4}$ Union College, USA \\ ${ }^{5}$ University of Salamanca, Spain
}

Article history

Received: 20-03-2017

Revised: 24-03-2017

Accepted: $30-03-2017$

Corresponding Author: Florian Ion T. Petrescu ARoTMM-IFToMM,

Bucharest Polytechnic

University, Bucharest, (CE)

Romania

Email: scipub02@gmail.com

\begin{abstract}
This paper presents and treats (in an original way) the specific elements of the structures of robotic solid mobile anthropomorphic type. Are "placed on the wallpaper", the geometry and kinematics of the anthropomorphic robotic solid systems, in an original vision of the authors. One presents the inverse kinematics of anthropomorphic systems, with mechanical elements and points: Geometry, cinematic, positions, displacements, velocities and accelerations. They will be presented further two methods (as the most representatives): First one the method trigonometric and second one the geometric method.
\end{abstract}

Keywords: Anthropomorphic Robots, Direct Kinematic, Inverse Kinematic, 3R Systems, Velocities, Accelerations

\section{Introduction}

Today, anthropomorphic structures are used more and more in almost all the fields of industrial. Robotic structures have emerged from the need for automation and robotics of the industrial processes. The first industrial robots were called upon by the heavy industry and in particular by the automobiles industry. The automotive industry not only has requested the appearance of the industrial robots but even their subsequent development (Angeles, 1989; Atkenson et al., 1986; Avallone and Baumeister, 1996; Baili, 2003; Baron and Angeles, 1998; Borrel and Liegeois, 1986; Burdick, 1988).

The most used were and have remained, the robots anthropomorphic, because they are more easily designed, built, maintained, are easily to handle, more dynamics, robust, economic and in general they have a broadly working area. The structures of the solid anthropomorphic robots are made up of elements and the couples of rotation, to which can add on an occasional basis and one or more couplers with translational moving. The couplers of rotation have been proven their effectiveness by moving them easier, more dynamic, step by step and especially being the most reliable. In general the couplers of rotation are moving more easily and more continuous, are actuated better and easier, control is less expensive and more reliable and programming the movements of rotation is also much simpler and more efficient (Ceccarelli, 1996; Choi et al., 2004; Denavit, 1964; Di Gregorio and Parenti-Castelli, 2002; Goldsmith, 2002; Grotjahn et al., 2004; Guegan and Khalil, 2002; Kim and Tsai, 2002; Lee and Sanderson, 2001; Liu and Kim, 2002).

All anthropomorphic structures are made up of a basic structure 3R (Fig. 1). Starting from the basic structure $3 R$ may build then various robots $4 R, 5 R$, $6 \mathrm{R}, 7 \mathrm{R}$..., by the addition of moving elements and the couplers. Regardless of how many degrees of mobility has a solid structure final mobile, the basis for the thing is always represented by the solid structure $3 R$ shown in Fig. 1. For this reason, the calculations presented in this study will be developed for the basic structure 3R. The structure in Fig. 1 consists of three moving elements linked together by the couplers of rotation. It is a spatial structure, with a large area of movement (working). 


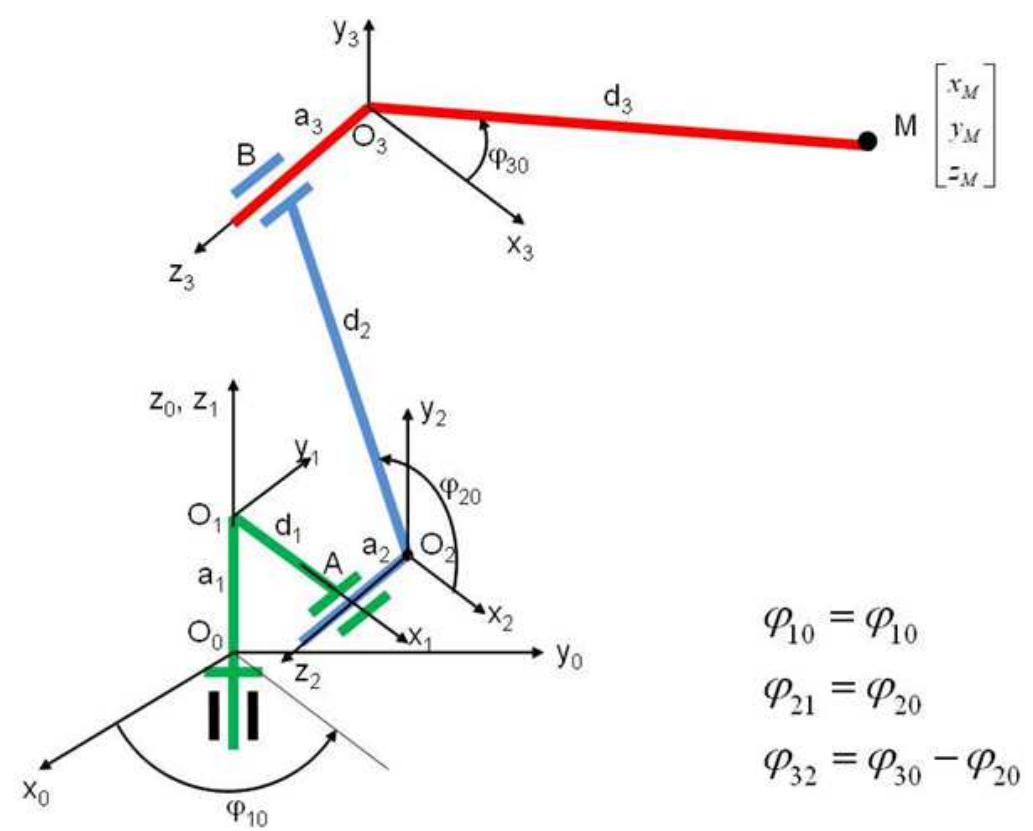

Fig. 1. Schema geometry-cinematic of a structure 3R modern (anthropomorphic)

The platform (system) as shown in Fig. 1 has three degree of mobility, carried out by three actuators (electric motors). The first electric motor drives the entire system in a rotating movement around a vertical shaft $\mathrm{O}_{0} \mathrm{Z}_{0}$. The engine (actuator) number 1 is mounted on the fixed element (frame 0) and causes the mobile element 1 in a rotation movement around a vertical shaft $\mathrm{O}_{0} \mathrm{O}_{1}$. On the mobile element 1 are constructed then all the other elements (components) of the system.

It follows a kinematic chain plan (vertical), composed of two elements in movement and two couplers cinematic engines. It is about the kinematic elements in movement 2 and 3, the assembly 2-3 being moved by the actuator of the second fitted in the coupler A, fixed on the element 1. Therefore the second electric motor attached to the component 1 will drive the element 2 in rotational motion relative to the item 1 and at the same time it will move the entire kinematic chain 2-3. The last actuator (electric motor) fixed by item 2, in B, will turn up the item 3 (relative in relation to the 2).

The rotation of the $\varphi_{10}$ carried out by the first actuator, is and relative (between items 1 and 0 ) and absolute (between items 1 and 0 ).

The rotation of the $\varphi_{20}$ carried out by the second actuator, is and relative rotation (between elements 2 and 1) and absolute (between items 2 and 0) due to the arrangement of the system.

The rotation of the $\theta=\varphi_{32}$ carried out by the third actuator, is only a relative rotation (between elements 3 and 2) and the corresponding absolute rotation (between items 3 and 0 ) is a function of the $\theta=\varphi_{32}$ and $\varphi_{20}$ (Aversa et al., 2016a; 2016b; 2016c; 2016d; 2016e; 2016f).
The kinematic chain 2-3 (consisting of kinematic elements in movement 2 and 3 ) is a kinematic chain in plan, which fall within a single plan or in one composed of several plane parallel. It is a system kinematic special, which may be studied separately. Then the element 1 (which it drives the kinematic chain 2-3) will be considered as coupler, kinematic couplers engines $\mathrm{A}\left(\mathrm{O}_{2}\right)$ and $\mathrm{B}\left(\mathrm{O}_{3}\right)$ becoming, the first fixed coupler and the second mobile coupler, both being kinematic couplers $\mathrm{C}_{5}$, of rotation.

For the determination of the extent of mobility of the kinematic chain (in plane) 2-3, shall apply to the structural formula given by the relationship (1), where $\mathrm{m}$ is the total number of moving parts of the kinematic chain plan; in our case $\mathrm{m}=2$ (as we are talking about the two kinematic elements in movement noted with 2 and 3 respectively) and the $\mathrm{C}_{5}$ represents the number kinematic couplers of the fifth-class, in this case $\mathrm{C}_{5}=2$ (as we are talking about the couplers $\mathrm{A}$ and $\mathrm{B}$ or $\mathrm{O}_{2}$ and $\mathrm{O}_{3}$ ):

$M_{3}=3 \cdot m-2 \cdot C_{5}=3 \cdot 2-2 \cdot 2=6-4=2$

The kinematic chain 2-3 having to the degree of mobility 2 , must be actuated by two motors. It is preferred that those two actuators to be two electric motors, direct current, or alternately.

Alternatively the drive of motion may be done and with engines hydraulic, pneumatic, sonic, etc. Structural diagram of the plan kinematic chain 2-3 (Fig. 2) resembles with its kinematic schema.

The driver element 2 is linked to the element 1 considered fixed, through the drive coupler $\mathrm{O}_{2}$ and driver element 3 is linked to the mobile element 2 through the drive coupler $\mathrm{O}_{3}$. 


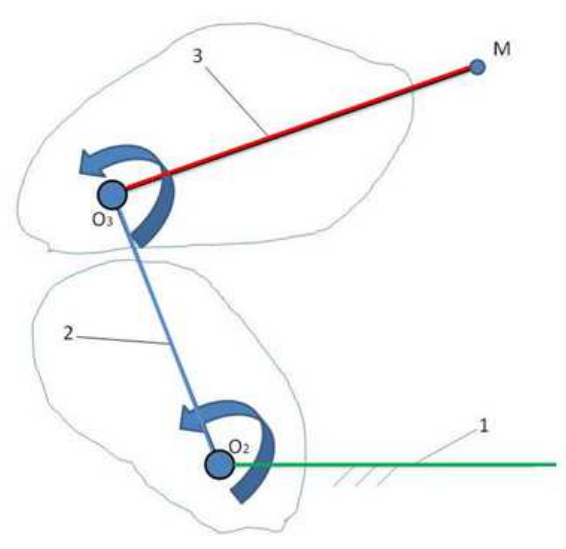

Fig. 2. Structural diagram of the kinematic chain plan 2-3 linked to item 1 considered fixed

As a result there is the kinematic chain open with two degree of mobility, carried out by the two actuators, i.e., the two electric motors, mounted on the motor kinematic couplers $\mathrm{A}$ and $\mathrm{B}$ or $\mathrm{O}_{2}$ in question $\mathrm{O}_{3}$ (Lorell et al., 2003; Merlet, 2000; Miller, 2004; Petrescu et al., 2009; Tsai, 2000).

\section{Direct Kinematic of the Plan Chain 2-3}

In Fig. 3 can be tracked cinematic diagram of the chain plan 2-3 open. In direct cinematic are known cinematic parameters $\varphi_{20}$ and $\varphi_{30}$ and must be determined by the analytical calculation the parameters $x_{M}$ and $y_{M}$, which represents the scalar coordinates of the point $M$ (endeffector $M$ ).

Are projected the vectors $d_{2}$ and $d_{3}$ on the Cartesian axis system considered fixed, $x O y$ identical with the $x_{2} \mathrm{O}_{2} y_{2}$, to obtain the system of equations scaling (2):

$$
\left\{\begin{array}{l}
x_{2 M} \equiv x_{M}=x_{O_{3}}+x_{3 M} \\
=d_{2} \cdot \cos \phi_{20}+d_{3} \cdot \cos \phi_{30}=d \cdot \cos \phi \\
y_{2 M} \equiv y_{M}=y_{O_{3}}+y_{3 M} \\
=d_{2} \cdot \sin \phi_{20}+d_{3} \cdot \sin \phi_{30}=d \cdot \sin \phi
\end{array}\right.
$$

After shall be determined in the form of Cartesian coordinates of the $M$ using the relations given by the system (2), may be obtained immediately and the $\varphi$ angle parameters using the relations estab-lished in the framework of the system (3):

$$
\left\{\begin{array}{l}
d^{2}=x_{M}^{2}+y_{M}^{2} \\
d=\sqrt{x_{M}^{2}+y_{M}^{2}} \\
\cos \phi=\frac{x_{M}}{d}=\frac{x_{M}}{\sqrt{x_{M}^{2}+y_{M}^{2}}} \\
\sin \phi=\frac{y_{M}}{d}=\frac{y_{M}}{\sqrt{x_{M}^{2}+y_{M}^{2}}} \\
\phi=\operatorname{sign}(\sin \phi) \cdot \arccos (\cos \phi)
\end{array}\right.
$$

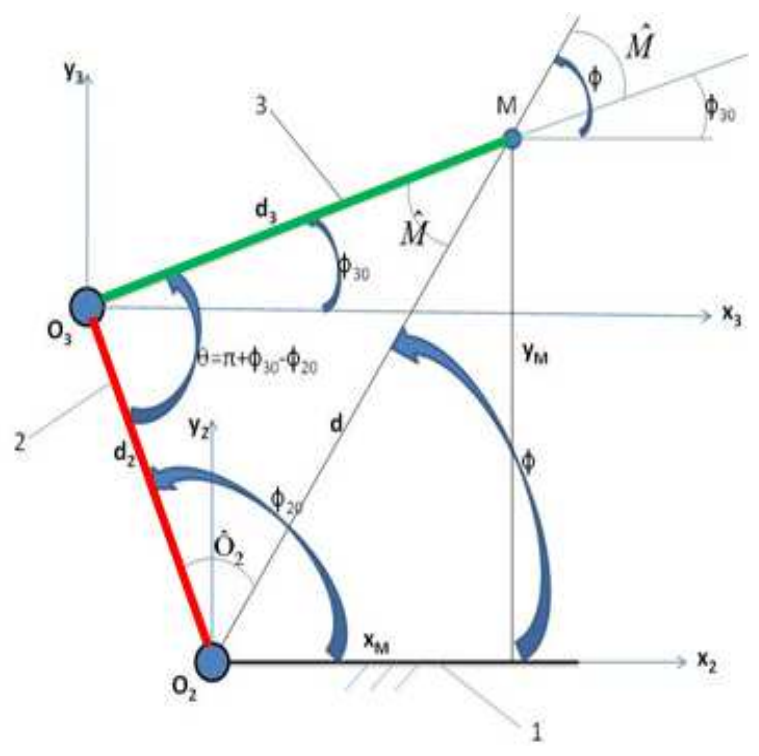

Fig. 3. Kinematic diagram of the kinematic chain plan 2-3 linked to item 1 considered fixed

The system (2) is written in a more concise manner in the form (4) which is derived with time to become the velocities system (5), which being derived with time generates in turn the system of accelerations (6):

$$
\begin{aligned}
& \left\{\begin{array}{l}
x_{M}=d_{2} \cdot \cos \phi_{20}+d_{3} \cdot \cos \phi_{30} \\
=d_{2} \cdot \cos \phi_{20}+d_{3} \cdot \cos \left(\theta+\phi_{20}-\pi\right) \\
y_{M}=d_{2} \cdot \sin \phi_{20}+d_{3} \cdot \sin \phi_{30} \\
=d_{2} \cdot \sin \phi_{20}+d_{3} \cdot \sin \left(\theta+\phi_{20}-\pi\right)
\end{array}\right. \\
& \left\{\begin{array}{l}
v_{M}^{x} \equiv \dot{x}_{M}=-d_{2} \cdot \sin \phi_{20} \cdot \omega_{20}-d_{3} \cdot \sin \phi_{30} \cdot \omega_{30} \\
=-d_{2} \cdot \sin \phi_{20} \cdot \omega_{20}-d_{3} \cdot \sin \phi_{30} \cdot\left(\dot{\theta}+\omega_{20}\right) \\
v_{M}^{y} \equiv \dot{y}_{M}=d_{2} \cdot \cos \phi_{20} \cdot \omega_{20}+d_{3} \cdot \cos \phi_{30} \cdot \omega_{30} \\
=d_{2} \cdot \cos \phi_{20} \cdot \omega_{20}+d_{3} \cdot \cos \phi_{30} \cdot\left(\dot{\theta}+\omega_{20}\right)
\end{array}\right. \\
& \left\{\begin{array}{l}
a_{M}^{x} \equiv \ddot{x}_{M}=-d_{2} \cdot \cos \phi_{20} \cdot \omega_{20}^{2}-d_{3} \cdot \cos \phi_{30} \cdot \omega_{30}^{2} \\
=-d_{2} \cdot \cos \phi_{20} \cdot \omega_{20}^{2}-d_{3} \cdot \cos \phi_{30} \cdot\left(\dot{\theta}+\omega_{20}\right)^{2} \\
a_{M}^{y} \equiv \ddot{y}_{M}=-d_{2} \cdot \sin \phi_{20} \cdot \omega_{20}^{2}-d_{3} \cdot \sin \phi_{30} \cdot \omega_{30}^{2} \\
=-d_{2} \cdot \sin \phi_{20} \cdot \omega_{20}^{2}-d_{3} \cdot \sin \phi_{30} \cdot\left(\dot{\theta}+\omega_{20}\right)^{2}
\end{array}\right.
\end{aligned}
$$

Remark: The rotation speeds of the actuators were considered to be constant (relations 7):

$$
\begin{aligned}
\dot{\phi}_{20} & =\omega_{20}=c t ; \quad \dot{\theta}=c t \Rightarrow s i \quad \omega_{30}=c t . \\
\varepsilon_{20} & =\ddot{\theta}=\varepsilon_{30}=0 .
\end{aligned}
$$

The relations (3) shall be derived and they and get the velocities (8) and the accelerations (9): 


$$
\left\{\begin{array}{l}
d^{2}=x_{M}^{2}+y_{M}^{2} \\
2 \cdot d \cdot \dot{d}=2 \cdot x_{M} \cdot \dot{x}_{M}+2 \cdot y_{M} \cdot \dot{y}_{M} \\
d \cdot \dot{d}=x_{M} \cdot \dot{x}_{M}+y_{M} \cdot \dot{y}_{M} \\
\dot{d}=\frac{x_{M} \cdot \dot{x}_{M}+y_{M} \cdot \dot{y}_{M}}{d} \\
d \cdot \cos \phi=x_{M} \\
d \cdot \sin \phi=y_{M} \\
\dot{d} \cdot \cos \phi-d \cdot \sin \phi \cdot \dot{\phi}=\dot{x}_{M} \mid \cdot(-\sin \phi) \\
\dot{d} \cdot \sin \phi+d \cdot \cos \phi \cdot \dot{\phi}=\dot{y}_{M} \mid \cdot(\cos \phi) \\
\frac{\dot{x} \dot{x}_{M} \cdot(-\sin \phi)+\dot{y}_{M} \cdot(\cos \phi)}{d \cdot \sin \phi} \\
\dot{\phi}=\frac{\dot{y}_{M} \cdot \cos \phi-\dot{x}_{M} \cdot \sin }{d=\frac{x_{M} \cdot \dot{x}_{M}+y_{M} \cdot \dot{y}_{M}}{d}}
\end{array}\right.
$$

$$
\left\{\begin{array}{l}
d^{2}=x_{M}^{2}+y_{M}^{2} \\
2 \cdot d \cdot \dot{d}=2 \cdot x_{M} \cdot \dot{x}_{M}+2 \cdot y_{M} \cdot \dot{y}_{M} \\
d \cdot \dot{d}=x_{M} \cdot \dot{x}_{M}+y_{M} \cdot \dot{y}_{M} \\
\dot{d}^{2}+d \cdot \ddot{d}=\dot{x}_{M}^{2}+x_{M} \cdot \ddot{x}_{M}+\dot{y}_{M}^{2}+y_{M} \cdot \ddot{y}_{M} \\
\ddot{d}=\frac{\dot{x}_{M}^{2}+x_{M} \cdot \ddot{x}_{M}+\dot{y}_{M}^{2}+y_{M} \cdot \ddot{y}_{M}-\dot{d}^{2}}{d} \\
d \cdot \cos \phi=x_{M} \\
d \cdot \sin \phi=y_{M} \\
\dot{d} \cdot \cos \phi-d \cdot \sin \phi \cdot \dot{\phi}=\dot{x}_{M} \mid \cdot(-\sin \phi) \\
\dot{d} \cdot \sin \phi+d \cdot \cos \phi \cdot \dot{\phi}=\dot{y}_{M} \mid \cdot(\cos \phi) \\
\frac{d \cdot \dot{\phi}=-\dot{x}_{M} \cdot \sin \phi+\dot{y}_{M} \cdot \cos \phi}{\dot{d} \cdot \dot{\phi}+d \cdot \ddot{\phi}=\ddot{y}_{M} \cdot \cos \phi-\dot{y}_{M} \cdot \sin \phi \cdot \dot{\phi}} \\
-\ddot{x}_{M} \cdot \sin \phi-\dot{x}_{M} \cdot \cos \phi \cdot \dot{\phi} \\
\ddot{\phi} \\
=\frac{\ddot{y}_{M} \cdot \cos \phi-\ddot{x}_{M} \cdot \sin \phi-\dot{y}_{M} \cdot \sin \phi \cdot \dot{\phi}-\dot{x}_{M} \cdot \cos \phi \cdot \dot{\phi}-\dot{d} \cdot \dot{\phi}}{d} \\
\frac{\dot{x}_{M}^{2}+x_{M} \cdot \ddot{x}_{M}+\dot{y}_{M}^{2}+y_{M} \cdot \ddot{y}_{M}-\dot{d}^{2}}{d}
\end{array}\right.
$$

The following will determine the positions, speeds and accelerations, according to the positions of the scales point $O_{3}$. It starts from the scalar coordinates of the point $\mathrm{O}_{3}$ (the relationships 10 ):

$$
\left\{\begin{array}{l}
x_{O_{3}}=d_{2} \cdot \cos \phi_{20} \\
y_{O_{3}}=d_{2} \cdot \sin \phi_{20}
\end{array}\right.
$$

Is then determined scaling speeds and accelerations of the point $O_{3}$, by differentiation of the system (10), in which shall be replaced after derivation the products d.cos or d.sin with the respective positions, $x_{\mathrm{O}_{3}}$ or $y_{\mathrm{O} 3}$, which become (in this way) variables (see relations 11 and 12 ):

$$
\begin{array}{r}
\left\{\begin{array}{l}
\dot{x}_{O_{3}}=-d_{2} \cdot \sin \phi_{20} \cdot \omega_{20}=-y_{O_{3}} \cdot \omega_{20} \\
\dot{y}_{O_{3}}=d_{2} \cdot \cos \phi_{20} \cdot \omega_{20}=x_{O_{3}} \cdot \omega_{20}
\end{array}\right. \\
\left\{\begin{array}{l}
\ddot{O}_{O_{3}}=-d_{2} \cdot \cos \phi_{20} \cdot \omega_{20}^{2}=-x_{O_{3}} \cdot \omega_{20}^{2} \\
\ddot{y}_{O_{3}}=-d_{2} \cdot \sin \phi_{20} \cdot \omega_{20}^{2}=-y_{O_{3}} \cdot \omega_{20}^{2}
\end{array}\right.
\end{array}
$$

Had been put in evidence in this mode the scalar speeds and accelerations of point $O_{3}$ according to their original positions (scaling) and absolute angular speed of the element 2.

The angular speed was considered to be constant. The technique of the determination of the velocities and acceleration depending on the positions, is extremely useful in the study of the dynamics of the system, of the vibrations and noise caused by the respective system.

This technique is common in the study of vibration system. Known the vibrations of scalar positions of point $\mathrm{O}_{3}$ and then easily determine the vibration velocities and accelerations that point and other points of the system all the functions of positions scaling known point $\mathrm{O}_{3}$.

Also by this technique can calculate local noise levels at various points in the system and the overall level of noise generated, with a precision good enough, compared with experimental measurements obtained with adequate equipment.

Absolute velocity of $O_{3}$ point (speed module) is given by relation (13) and the acceleration of $O_{3}$ can be written with expression (14):

$$
\left\{\begin{array}{l}
v_{O_{3}}=\sqrt{\dot{x}_{O_{3}}^{2}+\dot{y}_{O_{3}}^{2}} \\
=\sqrt{d_{2}^{2} \cdot \omega_{20}^{2} \cdot \sin ^{2} \phi_{20}+d_{2}^{2} \cdot \omega_{20}^{2} \cdot \cos ^{2} \phi_{20}} \\
=\sqrt{d_{2}^{2} \cdot \omega_{20}^{2}}=d_{2} \cdot \omega_{20}
\end{array}\right.
$$

$$
\left\{\begin{array}{l}
a_{O_{3}}=\sqrt{\ddot{x}_{O_{3}}^{2}+\ddot{y}_{O_{3}}^{2}} \\
=\sqrt{d_{2}^{2} \cdot \omega_{20}^{4} \cdot \cos ^{2} \phi_{20}+d_{2}^{2} \cdot \omega_{20}^{4} \cdot \sin ^{2} \phi_{20}} \\
=\sqrt{d_{2}^{2} \cdot \omega_{20}^{4}}=d_{2} \cdot \omega_{20}^{2}
\end{array}\right.
$$

In the following will determine the cinematic scalar parameters of the point $\mathrm{M}$, endeffector, depending and on the parameters of the position of points $O_{3}$ and $M$ (the systems of relations $15-17)$ : 


$$
\begin{aligned}
& \left\{\begin{array}{l}
x_{M}=x_{O_{3}}+d_{3} \cdot \cos \phi_{30} \\
y_{M}=y_{O_{3}}+d_{3} \cdot \sin \phi_{30} \\
d_{3} \cdot \cos \phi_{30}=x_{M}-x_{O_{3}} \\
d_{3} \cdot \sin \phi_{30}=y_{M}-y_{O_{3}}
\end{array}\right. \\
& \left\{\begin{array}{l}
\dot{x}_{M}=\dot{x}_{O_{3}}-d_{3} \cdot \sin \phi_{30} \cdot \dot{\phi}_{30} \\
=-y_{O_{3}} \cdot \omega_{20}+\left(y_{O_{3}}-y_{M}\right) \cdot\left(\omega_{20}+\dot{\theta}\right)
\end{array}\right. \\
& \left\{\begin{array}{l}
d^{2}=x_{M}^{2}+y_{M}^{2} ; d=\sqrt{x_{M}^{2}+y_{M}^{2}} \\
\cos \phi=\frac{x_{M}}{d}=\frac{x_{M}}{\sqrt{x_{M}^{2}+y_{M}^{2}}} ; \\
\sin \phi=\frac{y_{M}}{d}=\frac{y_{M}}{\sqrt{x_{M}^{2}+y_{M}^{2}}} \\
\phi=\operatorname{semn}(\sin \phi) \cdot \arccos (\cos \phi)
\end{array}\right.
\end{aligned}
$$$$
=y_{O_{3}} \cdot \dot{\theta}-y_{M} \cdot\left(\omega_{20}+\dot{\theta}\right)
$$$$
=\left(y_{O_{3}}-y_{M}\right) \cdot \dot{\theta}-y_{M} \cdot \omega_{20}
$$$$
\dot{y}_{M}=\dot{y}_{O_{3}}+d_{3} \cdot \cos \phi_{30} \cdot \dot{\phi}_{30}
$$$$
=x_{O_{3}} \cdot \omega_{20}+\left(x_{M}-x_{O_{3}}\right) \cdot\left(\omega_{20}+\dot{\theta}\right)
$$$$
=x_{M} \cdot\left(\omega_{20}+\dot{\theta}\right)-x_{O_{3}} \cdot \dot{\theta}
$$$$
=\left(x_{M}-x_{O_{3}}\right) \cdot \dot{\theta}+x_{M} \cdot \omega_{20}
$$$$
\dot{y}_{O_{3}}-\dot{y}_{M}=-d_{3} \cdot \cos \phi_{30} \cdot\left(\omega_{20}+\dot{\theta}\right)
$$$$
\dot{x}_{M}-\dot{x}_{O_{3}}=-d_{3} \cdot \sin \phi_{30} \cdot\left(\omega_{20}+\dot{\theta}\right)
$$$$
\left(\ddot{x}_{M}=\left(\dot{y}_{O_{3}}-\dot{y}_{M}\right) \cdot \dot{\theta}-\dot{y}_{M} \cdot \omega_{20}\right.
$$$$
\ddot{y}_{M}=\left(\dot{x}_{M}-\dot{x}_{O_{3}}\right) \cdot \dot{\theta}+\dot{x}_{M} \cdot \omega_{20}
$$$$
\dot{y}_{O_{3}}-\dot{y}_{M}=\left(x_{O_{3}}-x_{M}\right) \cdot\left(\omega_{20}+\dot{\theta}\right)
$$$$
\dot{x}_{M}-\dot{x}_{O_{3}}=\left(y_{O_{3}}-y_{M}\right) \cdot\left(\omega_{20}+\dot{\theta}\right)
$$$$
\ddot{x}_{M}=\left(x_{O_{3}}-x_{M}\right) \text {. }
$$$$
\cdot\left(\omega_{20}+\dot{\theta}\right) \cdot \dot{\theta}+\left(x_{O_{3}}-x_{M}\right) \cdot \dot{\theta} \cdot \omega_{20}-x_{M} \cdot \omega_{20}^{2}
$$$$
\ddot{y}_{M}=\left(y_{O_{3}}-y_{M}\right) \text {. }
$$$$
\cdot\left(\omega_{20}+\dot{\theta}\right) \cdot \dot{\theta}+\left(y_{O_{3}}-y_{M}\right) \cdot \dot{\theta} \cdot \omega_{20}-y_{M} \cdot \omega_{20}^{2}
$$$$
\ddot{x}_{M}=2 \cdot\left(x_{O_{3}}-x_{M}\right) \cdot \dot{\theta} \cdot \omega_{20}
$$$$
+\left(x_{O_{3}}-x_{M}\right) \cdot \dot{\theta}^{2}-x_{M} \cdot \omega_{20}^{2}
$$$$
\ddot{y}_{M}=2 \cdot\left(y_{O_{3}}-y_{M}\right) \cdot \dot{\theta} \cdot \omega_{20}
$$$$
+\left(y_{O_{3}}-y_{M}\right) \cdot \dot{\theta}^{2}-y_{M} \cdot \omega_{20}^{2}
$$$$
\ddot{x}_{M}=\left(x_{O_{3}}-x_{M}\right) \cdot\left(2 \cdot \dot{\theta} \cdot \omega_{20}+\dot{\theta}^{2}\right)-x_{M} \cdot \omega_{20}^{2}
$$$$
\ddot{y}_{M}=\left(y_{O_{3}}-y_{M}\right) \cdot\left(2 \cdot \dot{\theta} \cdot \omega_{20}+\dot{\theta}^{2}\right)-y_{M} \cdot \omega_{20}^{2}
$$$$
\ddot{x}_{M}=\left(x_{O_{3}}-x_{M}\right) \cdot\left(\omega_{20}+\dot{\theta}\right)^{2}-x_{O_{3}} \cdot \omega_{20}^{2}
$$$$
\ddot{y}_{M}=\left(y_{O_{3}}-y_{M}\right) \cdot\left(\omega_{20}+\dot{\theta}\right)^{2}-y_{O_{3}} \cdot \omega_{20}^{2}
$$

\section{Inverse Kinematic of the Plan Chain 2-3}

In Fig. 3 can be tracked cinematic diagram of the chain plan 2-3 open. In inverse cinematic are known the parameters $x_{M}$ and $y_{M}$ (which represent the scalar coordinates of the point $M$, endeffector $M$ ), imposed parameters and must be determined by the analytical calculations the cinematic parameters $\varphi_{20}$ and $\varphi_{30}$. Determine first the intermediate parameters $d$ and $\varphi$ with relations (18):
In triangle certain $\mathrm{O}_{2} \mathrm{O}_{3} \mathrm{M}$ know the lengths of the three sides, $d^{2}, d^{3}$ (constant) and $d$ (variable) so that it can be determined depending on the lengths of the sides all other elements of the triangle, specifically its angles and trigonometric functions of their (particularly interest us sin and cos). You can use various methods and they will be presented further two (as the most representatives): Method trigonometric and geometric method.

\section{Trigonometric Method, Determining Positions}

Writing of positions scaling Equations 19:

$$
\left\{\begin{array}{l}
d_{2} \cdot \cos \phi_{20}+d_{3} \cdot \cos \phi_{30}=x_{M} \\
d_{2} \cdot \sin \phi_{20}+d_{3} \cdot \sin \phi_{30}=y_{M} \\
\cos ^{2} \phi_{20}+\sin ^{2} \phi_{20}=1 \\
\cos ^{2} \phi_{30}+\sin ^{2} \phi_{30}=1
\end{array}\right.
$$

The problem with these two equations scaling, trigonometric, with two unknowns $\left(\varphi_{20}\right.$ and $\left.\varphi_{30}\right)$ is that they transcend (they are trigonometric equations, transcendental, where $\varphi_{20}$ unknown does not appear directly but in the form $\cos \varphi_{20}$ and $\sin \varphi_{20}$, so in reality the two trigonometric equations no longer have only two unknowns but four: $\cos \varphi_{20}, \sin \varphi_{20}, \cos \varphi_{30}$ and $\left.\sin \varphi_{30}\right)$.

To solve the system we need more two equations, so that in the system (19) were also added more two trigonometric equations, exactly the core "golden" as they are more say, for the angle $\varphi_{20}$ and separate for the $\varphi_{30}$ angle. In order to solve the first two equations of the system (19) are written in the form (20).

$\left\{\begin{array}{l}d_{2} \cdot \cos \phi_{20}-x_{M}=-d_{3} \cdot \cos \phi_{30} \\ d_{2} \cdot \sin \phi_{20}-y_{M}=-d_{3} \cdot \sin \phi_{30}\end{array}\right.$

Each equation of the system (20) are squared, then added together both equations (squared) to obtain the equation of the form (21):

$$
\left\{\begin{array}{l}
d_{2}^{2} \cdot\left(\cos ^{2} \phi_{20}+\sin ^{2} \phi_{20}\right)+x_{M}^{2}+y_{M}^{2} \\
-2 \cdot d_{2} \cdot x_{M} \cdot \cos \phi_{20} \\
-2 \cdot d_{2} \cdot y_{M} \cdot \sin \phi_{20}=d_{3}^{2} \cdot\left(\cos ^{2} \phi_{30}+\sin ^{2} \phi_{30}\right)
\end{array}\right.
$$


Now is the time to use the two "equations gold" written at the end trigonometric system (2), with which Equation 21 becomes simplified expression (22):

$$
\left\{\begin{array}{l}
d_{2}^{2}+x_{M}^{2}+y_{M}^{2}-2 \cdot d_{2} \cdot x_{M} \cdot \cos \phi_{20} \\
-2 \cdot d_{2} \cdot y_{M} \cdot \sin \phi_{20}=d_{3}^{2}
\end{array}\right.
$$

Arrange the terms of the Equation 22 in the most convenient form (23).

$$
\left\{\begin{array}{l}
d_{2}^{2}-d_{3}^{2}+x_{M}^{2}+y_{M}^{2} \\
=2 \cdot d_{2} \cdot\left(x_{M} \cdot \cos \phi_{20}+y_{M} \cdot \sin \phi_{20}\right)
\end{array}\right.
$$

Divide the Equation 23 with $2 . d_{2}$ and follows a new form (24):

$$
x_{M} \cdot \cos \phi_{20}+y_{M} \cdot \sin \phi_{20}=\frac{d_{2}^{2}-d_{3}^{2}+x_{M}^{2}+y_{M}^{2}}{2 \cdot d_{2}}
$$

Observing Fig. 2, from system (18) may be explicit the relation (25):

$$
x_{M}^{2}+y_{M}^{2}=d^{2}
$$

Insert (25) to (24) and amplified fraction the right to $\mathrm{d}$ so that the expression (24) takes the form (26) a more useful form:

$$
x_{M} \cdot \cos \phi_{20}+y_{M} \cdot \sin \phi_{20}=\frac{d_{2}^{2}+d^{2}-d_{3}^{2}}{2 \cdot d_{2} \cdot d} \cdot d
$$

Now, one can enter the expression cosine angle $O_{2}$, according to some $\mathrm{O}_{2} \mathrm{O}_{3} M$ triangle (27):

$$
\cos \hat{O}_{2}=\frac{d_{2}^{2}+d^{2}-d_{3}^{2}}{2 \cdot d_{2} \cdot d}
$$

Using the expression (27) Equation 26 becomes simplified (28):

$$
x_{M} \cdot \cos \phi_{20}-d \cdot \cos \hat{O}_{2}=-y_{M} \cdot \sin \phi_{20}
$$

Needs to remove $\sin \varphi_{20}$, for which we isolated the term in sin and rose squared Equation 28 for using the equation gold trigonometric angle $\varphi_{20}$ to transform sin in cosine, equation becoming one of the second degree in $\cos \varphi_{20}$. After squaring (28) becomes (29):

$$
\left\{\begin{array}{l}
x_{M}^{2} \cdot \cos ^{2} \phi_{20}+d^{2} \cdot \cos ^{2} \hat{O}_{2} \\
-2 \cdot d \cdot x_{M} \cdot \cos \hat{O}_{2} \cdot \cos \phi_{20}=y_{M}^{2} \cdot \sin ^{2} \phi_{20}
\end{array}\right.
$$

Use golden formula and expression (29) becomes (30) to be arranged conveniently by grouping terms of bringing to the form (31):

$$
\left\{\begin{array}{l}
x_{M}^{2} \cdot \cos ^{2} \phi_{20}+d^{2} \cdot \cos ^{2} \hat{O}_{2} \\
-2 \cdot d \cdot x_{M} \cdot \cos \hat{O}_{2} \cdot \cos \phi_{20}=y_{M}^{2}-y_{M}^{2} \cdot \cos ^{2} \phi_{20}
\end{array}\right.
$$

$\left\{\begin{array}{l}\left(x_{M}^{2}+y_{M}^{2}\right) \cdot \cos ^{2} \phi_{20}-2 \cdot d \cdot x_{M} \cdot \cos \hat{O}_{2} \cdot \cos \phi_{20} \\ -\left(y_{M}^{2}-d^{2} \cdot \cos ^{2} \hat{O}_{2}\right)=0\end{array}\right.$

Discriminant of Equation 31 in the second degree in cosine may be calculated by Equation 32:

$\left\{\begin{array}{l}\Delta=d^{2} \cdot x_{M}^{2} \cdot \cos ^{2} \hat{O}_{2}+d^{2} \cdot\left(y_{M}^{2}-d^{2} \cdot \cos ^{2} \hat{O}_{2}\right) \\ =d^{2} \cdot\left(x_{M}^{2} \cdot \cos ^{2} \hat{O}_{2}+y_{M}^{2}-d^{2} \cdot \cos ^{2} \hat{O}_{2}\right) \\ =d^{2} \cdot\left(y_{M}^{2}-y_{M}^{2} \cdot \cos ^{2} \hat{O}_{2}\right) \\ =d^{2} \cdot y_{M}^{2} \cdot\left(1-\cos ^{2} \hat{O}_{2}\right)=d^{2} \cdot y_{M}^{2} \cdot \sin ^{2} \hat{O}_{2}\end{array}\right.$

Radical second order of discriminant is expressed as (33):

$R=\sqrt{\Delta}=\sqrt{d^{2} \cdot y_{M}^{2} \cdot \sin ^{2} \hat{O}_{2}}=d \cdot y_{M} \cdot \sin \hat{O}_{2}$

Solutions to Equation 31 in the second degree in cosine are written in the form (34):

$\left\{\begin{array}{l}\cos \phi_{20_{1,2}}=\frac{d \cdot x_{M} \cdot \cos \hat{O}_{2} \mp d \cdot y_{M} \cdot \sin \hat{O}_{2}}{d^{2}} \\ =\frac{x_{M} \cdot \cos \hat{O}_{2} \mp y_{M} \cdot \sin \hat{O}_{2}}{d} \\ =\frac{x_{M}}{d} \cdot \cos \hat{O}_{2} \mp \frac{y_{M}}{d} \cdot \sin \hat{O}_{2}\end{array}\right.$

Further, in solutions (34) are replaced reports with corresponding trigonometric functions of angle $\varphi$, expressions (34) gaining form (35):

$\left\{\begin{array}{l}\cos \phi_{20_{1,2}}=\frac{x_{M}}{d} \cdot \cos \hat{O}_{2} \mp \frac{y_{M}}{d} \cdot \sin \hat{O}_{2} \\ =\cos \phi \cdot \cos \hat{O}_{2} \mp \sin \phi \cdot \sin \hat{O}_{2} \\ =\cos \left(\phi \pm \hat{O}_{2}\right) \\ \cos \phi_{20}=\cos \left(\phi \pm \hat{O}_{2}\right)\end{array}\right.$

One turns now to Equation 29 that it will be order in form (36) with a view to resolving them in sin. Equation 36 rises to the square and using trigonometric equation golden of angle $\varphi_{20}$, is obtained form (37):

$x_{M} \cdot \cos \phi_{20}=d \cdot \cos \hat{O}_{2}-y_{M} \cdot \sin \phi_{20}$ 


$$
\left\{\begin{array}{l}
x_{M}^{2} \cdot \cos ^{2} \phi_{20}=d^{2} \cdot \cos ^{2} \hat{O}_{2}+y_{M}^{2} \cdot \sin ^{2} \phi_{20} \\
-2 \cdot y_{M} \cdot d \cdot \cos \hat{O}_{2} \cdot \sin \phi_{20} \\
x_{M}^{2}-x_{M}^{2} \cdot \sin ^{2} \phi_{20}=d^{2} \cdot \cos ^{2} \hat{O}_{2}+y_{M}^{2} \cdot \sin ^{2} \phi_{20} \\
-2 \cdot y_{M} \cdot d \cdot \cos \hat{O}_{2} \cdot \sin \phi_{20} \\
\left(x_{M}^{2}+y_{M}^{2}\right) \cdot \sin ^{2} \phi_{20}-2 \cdot y_{M} \cdot d \cdot \cos \hat{O}_{2} \cdot \sin \phi_{20} \\
-\left(x_{M}^{2}-d^{2} \cdot \cos ^{2} \hat{O}_{2}\right)=0 \\
d^{2} \cdot \sin ^{2} \phi_{20}-2 \cdot y_{M} \cdot d \cdot \cos \hat{O}_{2} \cdot \sin \phi_{20} \\
-\left(x_{M}^{2}-d^{2} \cdot \cos ^{2} \hat{O}_{2}\right)=0
\end{array}\right.
$$

Discriminant Equation 37 in the second degree in cosine takes the form (38):

$$
\left\{\begin{array}{l}
\Delta=y_{M}^{2} \cdot d^{2} \cdot \cos ^{2} \hat{O}_{2}+d^{2} \cdot\left(x_{M}^{2}-d^{2} \cdot \cos ^{2} \hat{O}_{2}\right) \\
d^{2} \cdot\left(x_{M}^{2}+y_{M}^{2} \cdot \cos ^{2} \hat{O}_{2}-x_{M}^{2} \cdot \cos ^{2} \hat{O}_{2}-y_{M}^{2} \cdot \cos ^{2} \hat{O}_{2}\right) \\
=d^{2} \cdot\left(x_{M}^{2}-x_{M}^{2} \cdot \cos ^{2} \hat{O}_{2}\right)=d^{2} \cdot x_{M}^{2} \cdot \sin ^{2} \hat{O}_{2}
\end{array}\right.
$$

Solutions to Equation 36 are written now as (39):

$$
\left\{\begin{array}{l}
\sin \phi_{20}=\frac{y_{M} \cdot d \cdot \cos \hat{O}_{2} \pm x_{M} \cdot d \cdot \sin \hat{O}_{2}}{d^{2}} \\
=\frac{y_{M} \cdot \cos \hat{O}_{2} \pm x_{M} \cdot \sin \hat{O}_{2}}{d} \\
=\frac{y_{M}}{d} \cdot \cos \hat{O}_{2} \pm \frac{x_{M}}{d} \cdot \sin \hat{O}_{2} \\
=\sin \phi \cdot \cos \hat{O}_{2} \pm \cos \phi \cdot \sin \hat{O}_{2}=\sin \left(\phi \pm \hat{O}_{2}\right) \\
\sin \phi_{20}=\sin \left(\phi \pm \hat{O}_{2}\right)
\end{array}\right.
$$

From resulted relations (40) it can write the basic relationship (41):

$$
\begin{aligned}
& \left\{\begin{array}{l}
\cos \phi_{20}=\cos \left(\phi \pm \hat{O}_{2}\right) \\
\sin \phi_{20}=\sin \left(\phi \pm \hat{O}_{2}\right)
\end{array}\right. \\
& \phi_{20}=\phi \pm \hat{O}_{2}
\end{aligned}
$$

The procedure for determining the angle $\varphi_{30}$, starting again from the system (19), where the first two transcendental equations are rewritten as (42) in order to eliminate $\varphi_{20}$ angle this time:

$$
\left\{\begin{array}{l}
d_{2} \cdot \cos \phi_{20}=x_{M}-d_{3} \cdot \cos \phi_{30} \\
d_{2} \cdot \sin \phi_{20}=y_{M}-d_{3} \cdot \sin \phi_{30}
\end{array}\right.
$$

Raises the two Equation 42 squared and added together, resulting in the equation of the form (43), which is arranged in the most convenient forms (44) and (45):

$$
\begin{aligned}
& \left\{\begin{array}{l}
d_{2}^{2}=x_{M}^{2}+y_{M}^{2}+d_{3}^{2} \\
-2 \cdot d_{3} \cdot x_{M} \cdot \cos \phi_{30}-2 \cdot d_{3} \cdot y_{M} \cdot \sin \phi_{30}
\end{array}\right. \\
& x_{M} \cdot \cos \phi_{30}+y_{M} \cdot \sin \phi_{30}=d \cdot \frac{d^{2}+d_{3}^{2}-d_{2}^{2}}{2 \cdot d \cdot d_{3}} \\
& x_{M} \cdot \cos \phi_{30}+y_{M} \cdot \sin \phi_{30}=d \cdot \cos \hat{M}
\end{aligned}
$$

Need to determine first the cosine so that we isolate initially term in sin, Equation 45 with a special its form (46), which by squaring generates expression (47), an expression that arranges form (48):

$x_{M} \cdot \cos \phi_{30}-d \cdot \cos \hat{M}=-y_{M} \cdot \sin \phi_{30}$

$\left\{\begin{array}{l}x_{M}^{2} \cdot \cos ^{2} \phi_{30}+d^{2} \cdot \cos ^{2} \hat{M} \\ -2 \cdot d \cdot x_{M} \cdot \cos \hat{M} \cdot \cos \phi_{30}=y_{M}^{2}-y_{M}^{2} \cdot \cos ^{2} \phi_{30}\end{array}\right.$

$\left\{\begin{array}{l}d^{2} \cdot \cos ^{2} \phi_{30}-2 \cdot d \cdot x_{M} \cdot \cos \hat{M} \cdot \cos \phi_{30} \\ -\left(y_{M}^{2}-d^{2} \cdot \cos ^{2} \hat{M}\right)=0\end{array}\right.$

Equation 48 is a second degree equation in cosine with solutions given by expression (49):

$$
\left\{\begin{array}{l}
\cos \phi_{30}= \\
\frac{d \cdot x_{M} \cdot \cos \hat{M} \pm \sqrt{d^{2} \cdot x_{M}^{2} \cdot \cos ^{2} \hat{M}+d^{2} \cdot\left(y_{M}^{2}-d^{2} \cdot \cos ^{2} \hat{M}\right)}}{d^{2}} \\
=\frac{d \cdot x_{M} \cdot \cos \hat{M} \pm \sqrt{d^{2} \cdot y_{M}^{2} \cdot\left(1-\cos ^{2} \hat{M}\right)}}{d^{2}} \\
=\frac{d \cdot x_{M} \cdot \cos \hat{M} \pm d \cdot y_{M} \cdot \sin \hat{M}}{d^{2}} \\
=\frac{x_{M}}{d} \cdot \cos \hat{M} \pm \frac{y_{M}}{d} \cdot \sin \hat{M} \\
=\cos \phi \cdot \cos \hat{M} \pm \sin \phi \cdot \sin \hat{M}=\cos (\phi \mp \hat{M}) \\
\cos \phi_{30}=\cos (\phi \mp \hat{M})
\end{array}\right.
$$

Further write Equation 45 as (50), which is isolated this time cosine to eliminate it and then to determine the term $\sin$. The expression (52) is an equation of the second degree in sin, admitting the solutions given by (53):

$x_{M} \cdot \cos \phi_{30}=d \cdot \cos \hat{M}-y_{M} \cdot \sin \phi_{30}$

Equation 50 amounts to the square and obtain the equation of the form (51) suitable to be arranged in the form (52):

$\left\{\begin{array}{l}x_{M}^{2} \cdot\left(1-\sin ^{2} \phi_{30}\right)=d^{2} \cdot \cos ^{2} \hat{M} \\ +y_{M}^{2} \cdot \sin ^{2} \phi_{30}-2 \cdot y_{M} \cdot d \cdot \cos \hat{M} \cdot \sin \phi_{30}\end{array}\right.$ 


$$
\begin{aligned}
& \left\{\begin{array}{l}
d^{2} \cdot \sin ^{2} \phi_{30}-2 \cdot y_{M} \cdot d \cdot \cos \hat{M} \cdot \sin \phi_{30} \\
-\left(x_{M}^{2}-d^{2} \cdot \cos ^{2} \hat{M}\right)=0
\end{array}\right. \\
& \left\{\begin{array}{l}
\sin \phi_{30}= \\
\frac{d \cdot y_{M} \cdot \cos \hat{M} \mp \sqrt{d^{2} \cdot y_{M}^{2} \cdot \cos ^{2} \hat{M}+d^{2} \cdot\left(x_{M}^{2}-d^{2} \cdot \cos ^{2} \hat{M}\right)}}{d^{2}} \\
=\frac{d \cdot y_{M} \cdot \cos \hat{M} \mp \sqrt{d^{2} \cdot x_{M}^{2} \cdot\left(1-\cos ^{2} \hat{M}\right)}}{d^{2}} \\
=\frac{d \cdot y_{M} \cdot \cos \hat{M} \mp d \cdot x_{M} \cdot \sin \hat{M}}{d^{2}} \\
=\frac{y_{M}}{d} \cdot \cos \hat{M} \mp \frac{x_{M}}{d} \cdot \sin \hat{M} \\
=\sin \phi \cdot \cos \hat{M} \mp \cos \phi \cdot \sin \hat{M}=\sin (\phi \mp \hat{M}) \\
\sin \phi_{30}=\sin (\phi \mp \hat{M})
\end{array}\right.
\end{aligned}
$$

Then, are qualified only the relations (54) and (55):

$$
\begin{aligned}
& \left\{\begin{array}{l}
\cos \phi_{30}=\cos (\phi \mp \hat{M}) \\
\sin \phi_{30}=\sin (\phi \mp \hat{M})
\end{array}\right. \\
& \phi_{30}=\phi \mp \hat{M}
\end{aligned}
$$

\section{Trigonometric Method, Determining Velocities}

Starting from relations (56) required in the study of velocities in inverse kinematics:

$$
\left\{\begin{array}{l}
\dot{\phi}=\frac{\dot{y}_{M} \cdot \cos \phi-\dot{x}_{M} \cdot \sin \phi}{d} \\
\dot{d}=\frac{x_{M} \cdot \dot{x}_{M}+y_{M} \cdot \dot{y}_{M}}{d}
\end{array}\right.
$$

It starts from the relationship linking the cosine of the angle $\hat{O}_{2}$ of the triangle sides, which derives in relation to time and thus obtains the value $\dot{\hat{O}}_{2}$ written simply $\dot{O}_{2}$ (relations 57):

$$
\left\{\begin{array}{l}
2 \cdot d_{2} \cdot d \cdot \cos O_{2}=d_{2}^{2}-d_{3}^{2}+d^{2} \\
2 \cdot d_{2} \cdot \dot{d} \cdot \cos O_{2}-2 \cdot d_{2} \cdot d \cdot \sin O_{2} \cdot \dot{O}_{2} \\
=2 \cdot d \cdot \dot{d} \Rightarrow \dot{O}_{2}=\frac{d_{2} \cdot \dot{d} \cdot \cos O_{2}-d \cdot \dot{d}}{d_{2} \cdot d \cdot \sin O_{2}}
\end{array}\right.
$$

It derives the Equation 24 to give the angular velocity $\omega_{20} \equiv \dot{\phi}_{20} \quad$ (relation 58):

$\omega_{20} \equiv \dot{\phi}_{20}=\dot{\phi} \pm \dot{O}_{2}$
To determine $\omega_{20}$ (relationship 58) we need $\dot{\phi}$ to be calculated from (56) and $\dot{O}_{2}$ which is determined from (57). In turn $\dot{O}_{2}$ requires for its calculation $\dot{d}$ that calculates all from system (56). Inlet velocities $\dot{x}_{M}$ and $\dot{y}_{M}$ are known, are required as input, or choose convenient, or can be calculated based on criteria imposed. Similarly, one determines and the angular velocity $\omega_{30} \equiv \dot{\phi}_{30}$ (relation 59). It derives the equation (55) to obtain the angular velocity $\omega_{30} \equiv \dot{\phi}_{30}$ (expression 60). Then, $\dot{\phi}$ is calculated using the expression system known already (56) and $\dot{M}$ is determined from the system (59) and by the help of the system (56) which it causes and $\dot{d}$ :

$$
\begin{aligned}
& \left\{\begin{array}{l}
2 \cdot d_{3} \cdot d \cdot \cos M=d_{3}^{2}-d_{2}^{2}+d^{2} \\
2 \cdot d_{3} \cdot \dot{d} \cdot \cos M-2 \cdot d_{3} \cdot d \cdot \sin M \cdot \dot{M} \\
=2 \cdot d \cdot \dot{d} \Rightarrow \dot{M}=\frac{d_{3} \cdot \dot{d} \cdot \cos M-d \cdot \dot{d}}{d_{3} \cdot d \cdot \sin M}
\end{array}\right. \\
& \omega_{30} \equiv \dot{\phi}_{30}=\dot{\phi} \mp \dot{M}
\end{aligned}
$$

\section{Trigonometric Method, Determining Accelerations}

It starts from the relationships (61) required in the study accelerations in inverse kinematics. The relation of the system (57) derived a second time to time, gives the system (62):

$\left\{\begin{array}{l}\ddot{\phi}=\frac{\ddot{y}_{M} \cdot \cos \phi-\ddot{x}_{M} \cdot \sin \phi}{d} \\ +\frac{-\dot{y}_{M} \cdot \sin \phi \cdot \dot{\phi}-\dot{x}_{M} \cdot \cos \phi \cdot \dot{\phi}-\dot{d} \cdot \dot{\phi}}{d} \\ \ddot{d}=\frac{\dot{x}_{M}^{2}+x_{M} \cdot \ddot{x}_{M}+\dot{y}_{M}^{2}+y_{M} \cdot \ddot{y}_{M}-\dot{d}^{2}}{d}\end{array}\right.$

$\left\{\begin{array}{l}2 \cdot d_{2} \cdot d \cdot \cos O_{2}=d_{2}^{2}-d_{3}^{2}+d^{2} \\ 2 \cdot d_{2} \cdot \dot{d} \cdot \cos O_{2}-2 \cdot d_{2} \cdot d \cdot \sin O_{2} \cdot \dot{O}_{2}=2 \cdot d \cdot \dot{d} \\ \Rightarrow d_{2} \cdot d \cdot \sin O_{2} \cdot \dot{O}_{2}=d_{2} \cdot \dot{d} \cdot \cos O_{2}-d \cdot \dot{d} \\ \ddot{O}_{2}=\frac{\ddot{d} d_{2} \cos O_{2}-\ddot{d} d-2 \dot{d} d_{2} \sin O_{2} \cdot \dot{O}_{2}-d_{2} \cos O_{2} \cdot \dot{O}_{2}^{2}-\dot{d}^{2}}{d_{2} \cdot d \cdot \sin O_{2}}\end{array}\right.$

The following is derives the expression (58) to obtains expression (63), which generates angular acceleration absolute $\varepsilon_{2} \equiv \varepsilon_{20}$, that is calculated with $\ddot{\phi}$ out of the system (61) and with $\ddot{O}_{2}$ out of the system (45) and for the determination of $\ddot{O}_{2}$ longer needed $\ddot{d}$ removed all from relations (61): 


$$
\varepsilon_{2} \equiv \varepsilon_{20}=\dot{\omega}_{20} \equiv \ddot{\phi}_{20}=\ddot{\phi} \pm \ddot{O}_{2}
$$

Now it derives a second time (59) and obtains the system (64):

$$
\left\{\begin{array}{l}
2 \cdot d_{3} \cdot d \cdot \cos M=d_{3}^{2}-d_{2}^{2}+d^{2} \\
2 \cdot d_{3} \cdot \dot{d} \cdot \cos M-2 \cdot d_{3} \cdot d \cdot \sin M \cdot \dot{M}=2 \cdot d \cdot \dot{d} \\
\Rightarrow d_{3} \cdot d \cdot \sin M \cdot \dot{M}=d_{3} \cdot \dot{d} \cdot \cos M-d \cdot \dot{d} \\
\ddot{M}=\frac{\ddot{d} d_{3} \cos M-\ddot{d} d-2 \dot{d} d_{3} \sin M \cdot \dot{M}-d d_{3} \cos M \cdot \dot{M}^{2}-\dot{d}^{2}}{d_{3} \cdot d \cdot \sin M}
\end{array}\right.
$$

It derives again the time relation (60) and we obtain the expression (65) of the angular acceleration absolute $\varepsilon_{3} \equiv \varepsilon_{30}$ which is determined with $\ddot{\phi}$ and $\ddot{M}$ :

$$
\varepsilon_{3} \equiv \varepsilon_{30}=\dot{\omega}_{30} \equiv \ddot{\phi}_{30}=\ddot{\phi} \mp \ddot{M}
$$

\section{Geometric Method, Determining Positions}

It starts by writing positions equations, geometric (geometro-analytical) (66). Scaling coordinates $\left(x_{M}, y_{M}\right)$ of point $M$ (endeffector) are known and must be determined and scaling coordinates of the point $O_{3}$, which we denote by $(x, y)$. The relationships of the system (66) are obtained by writing the geometro-analytical equations of the circles, the rays $d_{3}$ and $d_{2}$ respectively:

$$
\left\{\begin{array}{l}
\left(x-x_{M}\right)^{2}+\left(y-y_{M}\right)^{2}=d_{3}^{2} \\
x^{2}+y^{2}=d_{2}^{2}
\end{array}\right.
$$

Release binomials of the first equation of the system, enter the second equation into the first, is also used and expression $d^{2}=x_{M}^{2}+y_{M}^{2}$, of amplify fraction with the factor convenient $d \cdot d_{2}$ and obtaining the final expression of system (67), which is written together with the equation of the second from system (66) in the new system (68) who needs to be addressed:

$$
\begin{aligned}
& \left\{\begin{array}{l}
x_{M} \cdot x+y_{M} \cdot y=\frac{d_{2}^{2}+d^{2}-d_{3}^{2}}{2} \\
x_{M} \cdot x+y_{M} \cdot y=d \cdot d_{2} \cdot \frac{d_{2}^{2}+d^{2}-d_{3}^{2}}{2 \cdot d \cdot d_{2}} \\
x_{M} \cdot x+y_{M} \cdot y=d \cdot d_{2} \cdot \cos O_{2}
\end{array}\right. \\
& \left\{\begin{array}{l}
x_{M} \cdot x+y_{M} \cdot y=d \cdot d_{2} \cdot \cos O_{2} \\
x^{2}+y^{2}=d_{2}^{2}
\end{array}\right.
\end{aligned}
$$

From the first equation of the system (68) explains the value of $y$, which rose and squared (69):

$$
\left\{\begin{array}{l}
y=\frac{d \cdot d_{2} \cdot \cos O_{2}-x_{M} \cdot x}{y_{M}} \\
y^{2}=\frac{d^{2} \cdot d_{2}^{2} \cdot \cos ^{2} O_{2}+x_{M}^{2} \cdot x^{2}-2 \cdot x_{M} \cdot d_{2} \cdot d \cdot \cos O_{2} \cdot x}{y_{M}^{2}}
\end{array}\right.
$$

The phrase 'second (69) is introduced into a second relationship of (68) to give Equation 70, which are arranged conveniently in the form (71):

$$
\left\{\begin{array}{l}
y_{M}^{2} \cdot x^{2}+d^{2} \cdot d_{2}^{2} \cdot \cos ^{2} O_{2}+x_{M}^{2} \cdot x^{2} \\
-2 \cdot x_{M} \cdot d_{2} \cdot d \cdot \cos O_{2} \cdot x-y_{M}^{2} \cdot d_{2}^{2}=0
\end{array}\right.
$$

$\left\{\begin{array}{l}d^{2} \cdot x^{2}-2 \cdot x_{M} \cdot d_{2} \cdot d \cdot \cos O_{2} \cdot x \\ -d_{2}^{2} \cdot\left(y_{M}^{2}-d^{2} \cdot \cos ^{2} O_{2}\right)=0\end{array}\right.$

Equation 71 is an equation of the second degree in $x$, which allows real solutions (72):

$$
\left\{\begin{array}{l}
x=\frac{x_{M} \cdot d_{2} \cdot d \cdot \cos O_{2}}{d^{2}} \mp \\
\frac{\sqrt{x_{M}^{2} \cdot d_{2}^{2} \cdot d^{2} \cdot \cos O_{2}+d^{2} \cdot d_{2}^{2} \cdot\left(y_{M}^{2}-d^{2} \cdot \cos ^{2} O_{2}\right)}}{d^{2}} \\
=\frac{x_{M} \cdot d_{2} \cdot d \cdot \cos O_{2} \mp d_{2} \cdot d \cdot y_{M} \cdot \sqrt{1-\cos ^{2} O_{2}}}{d^{2}} \\
=\frac{x_{M} \cdot d_{2} \cdot \cos O_{2} \mp d_{2} \cdot y_{M} \cdot \sqrt{\sin ^{2} O_{2}}}{d} \\
=\frac{x_{M} \cdot d_{2} \cdot \cos O_{2} \mp d_{2} \cdot y_{M} \cdot \sin O_{2}}{d} \\
=d_{2} \cdot\left(\frac{x_{M}}{d} \cdot \cos O_{2} \mp \frac{y_{M}}{d} \cdot \sin O_{2}\right) \\
=d_{2} \cdot\left(\cos \phi \cdot \cos O_{2} \mp \sin \phi \cdot \sin O_{2}\right) \\
=d_{2} \cdot \cos \left(\phi \pm O_{2}\right) \\
x=d_{2} \cdot \cos \left(\phi \pm O_{2}\right)
\end{array}\right.
$$

It further determines the unknown y by introducing value $x$ obtained from (72) in the first relationship of the system (69). One obtains the expression (73):

$$
\left\{\begin{array}{l}
y=\frac{d \cdot d_{2} \cdot \cos O_{2}-x_{M} \cdot d_{2} \cdot\left(\frac{x_{M}}{d} \cdot \cos O_{2} \mp \frac{y_{M}}{d} \cdot \sin O_{2}\right)}{y_{M}} \\
=\frac{d_{2} \cdot\left(\left(x_{M}^{2}+y_{M}^{2}\right) \cdot \cos O_{2}-x_{M}^{2} \cdot \cos O_{2} \pm x_{M} \cdot y_{M} \cdot \sin O_{2}\right)}{d \cdot y_{M}} \\
=d_{2} \cdot\left(\frac{y_{M}}{d} \cdot \cos O_{2} \pm \frac{x_{M}}{d} \cdot \sin O_{2}\right) \\
=d_{2} \cdot\left(\sin \phi \cdot \cos O_{2} \pm \cos \phi \cdot \sin O_{2}\right)=d_{2} \cdot \sin \left(\phi \pm O_{2}\right)
\end{array}\right.
$$


From (72) and (73) retain only the latest expression concentrated both in system (74):

$$
\left\{\begin{array}{l}
x=d_{2} \cdot \cos \left(\phi \pm O_{2}\right) \\
y=d_{2} \cdot \sin \left(\phi \pm O_{2}\right)
\end{array}\right.
$$

From Fig. 3 may write Equation 75:

$$
\left\{\begin{array}{l}
x=d_{2} \cdot \cos \phi_{20} \\
y=d_{2} \cdot \sin \phi_{20}
\end{array}\right.
$$

Comparing systems (74) and (75) the resulting system (76), from that is deducting direct relation (77):

$$
\begin{aligned}
& \left\{\begin{array}{l}
\cos \phi_{20}=\cos \left(\phi \pm O_{2}\right) \\
\sin \phi_{20}=\sin \left(\phi \pm O_{2}\right)
\end{array}\right. \\
& \phi_{20}=\phi \pm O_{2}
\end{aligned}
$$

\section{Geometric Method, Determining Velocities}

Starting from the positions system (66), which is derived a function of time and obtains the velocities system (78). The system (78) is rewritten in simplified form (79):

$$
\begin{aligned}
& \left\{\begin{array}{l}
2 \cdot\left(x-x_{M}\right) \cdot\left(\dot{x}-\dot{x}_{M}\right)+2 \cdot\left(y-y_{M}\right) \cdot\left(\dot{y}-\dot{y}_{M}\right)=0 \\
2 \cdot x \cdot \dot{x}+2 \cdot y \cdot \dot{y}=0
\end{array}\right. \\
& \left\{\begin{array}{l}
\left(x-x_{M}\right) \cdot \dot{x}+\left(y-y_{M}\right) \cdot \dot{y} \\
=\left(x-x_{M}\right) \cdot \dot{x}_{M}+\left(y-y_{M}\right) \cdot \dot{y}_{M} \\
x \cdot \dot{x}+y \cdot \dot{y}=0
\end{array}\right.
\end{aligned}
$$

In (79) break out the brackets and get the system (80):

$$
\left\{\begin{array}{l}
x \cdot \dot{x}+y \cdot \dot{y}-\left(x_{M} \cdot \dot{x}+y_{M} \cdot \dot{y}\right) \\
=\left(x-x_{M}\right) \cdot \dot{x}_{M}+\left(y-y_{M}\right) \cdot \dot{y}_{M} \\
x \cdot \dot{x}+y \cdot \dot{y}=0
\end{array}\right.
$$

The second relationship of system (80) is inserted in the first, then the first expression is multiplied by $(-1)$, so the system is simplified, receiving the form (81):

$$
\left\{\begin{array}{l}
x_{M} \cdot \dot{x}+y_{M} \cdot \dot{y}=\left(x_{M}-x\right) \cdot \dot{x}_{M}+\left(y_{M}-y\right) \cdot \dot{y}_{M} \\
x \cdot \dot{x}+y \cdot \dot{y}=0
\end{array}\right.
$$

The system (81) is solved in two steps.

In the first step is multiplied the first relation of the system $(81)$ by $(y)$ and the second by $\left(-y_{M}\right)$, after which the result expressions are gathered member by member to provide the following relation (82) in which it is explained $\dot{x}$ :

$$
\dot{x}=\frac{y \cdot\left[\left(x_{M}-x\right) \cdot \dot{x}_{M}+\left(y_{M}-y\right) \cdot \dot{y}_{M}\right]}{x_{M} \cdot y-y_{M} \cdot x}
$$

In step two we want to achieve $\dot{y}$ for which multiplies the first relationship of the system (81) by $(x)$ and the second with $\left(-x_{M}\right)$, gather relations obtained member with member and explains $\dot{y}$ resulting relationship (83):

$\dot{y}=\frac{-x \cdot\left[\left(x_{M}-x\right) \cdot \dot{x}_{M}+\left(y_{M}-y\right) \cdot \dot{y}_{M}\right]}{x_{M} \cdot y-y_{M} \cdot x}$

Relationships (82) and (83) are written confined within the system (84):

$$
\left\{\begin{array}{l}
\dot{x}=y \cdot h \\
\dot{y}=-x \cdot h \\
h=\frac{\left(x_{M}-x\right) \cdot \dot{x}_{M}+\left(y_{M}-y\right) \cdot \dot{y}_{M}}{x_{M} \cdot y-y_{M} \cdot x}
\end{array}\right.
$$

\section{Geometric Method, Determining Accelerations}

Starting from the velocities system (84), which is derived a function of time and obtains acceleration system (85). The system (85) is rewritten as (86):

$$
\begin{aligned}
& \ddot{x}=\dot{y} \cdot h+y \cdot \dot{h}=-x \cdot h^{2}+y \cdot \dot{h} \\
& \ddot{y}=-\dot{x} \cdot h-x \cdot \dot{h}=-y \cdot h^{2}-x \cdot \dot{h} \\
& h \cdot\left(x_{M} \cdot y-y_{M} \cdot x\right) \\
& =\left(x_{M}-x\right) \cdot \dot{x}_{M}+\left(y_{M}-y\right) \cdot \dot{y}_{M} \\
& \dot{h} \cdot\left(x_{M} \cdot y-y_{M} \cdot x\right) \\
& +h \cdot\left(\dot{x}_{M} \cdot y+x_{M} \cdot \dot{y}-\dot{y}_{M} \cdot x-y_{M} \cdot \dot{x}\right) \\
& =\left(\dot{x}_{M}-\dot{x}\right) \cdot \dot{x}_{M}+\left(x_{M}-x\right) \cdot \ddot{x}_{M} \\
& +\left(\dot{y}_{M}-\dot{y}\right) \cdot \dot{y}_{M}+\left(y_{M}-y\right) \cdot \ddot{y}_{M} \\
& \begin{array}{l}
\dot{h}=\frac{\left(\dot{x}_{M}-\dot{x}\right) \cdot \dot{x}_{M}+\left(x_{M}-x\right) \cdot \ddot{x}_{M}}{x_{M} \cdot y-y_{M} \cdot x} \\
+\frac{\left(\dot{y}_{M}-\dot{y}\right) \cdot \dot{y}_{M}+\left(y_{M}-y\right) \cdot \ddot{y}_{M}}{x_{M} \cdot y-y_{M} \cdot x} \\
-h \cdot \frac{\dot{x}_{M} \cdot y+x_{M} \cdot \dot{y}-\dot{y}_{M} \cdot x-y_{M} \cdot \dot{x}}{x_{M} \cdot y-y_{M} \cdot x}
\end{array} \\
& \left\{\begin{array}{l}
\ddot{x}=\dot{y} \cdot h+y \cdot \dot{h}=-x \cdot h^{2}+y \cdot \dot{h} \\
\ddot{y}=-\dot{x} \cdot h-x \cdot \dot{h}=-y \cdot h^{2}-x \cdot \dot{h} \\
\dot{h}=\frac{\left(\dot{x}_{M}-\dot{x}-y \cdot h\right) \cdot \dot{x}_{M}+\left(\dot{y}_{M}-\dot{y}+x \cdot h\right) \cdot \dot{y}_{M}}{x_{M} \cdot y-y_{M} \cdot x} \\
+\frac{\left(x_{M}-x\right) \cdot \ddot{x}_{M}+\left(y_{M}-y\right) \cdot \ddot{y}_{M}+y_{M} \cdot \dot{x} \cdot h-x_{M} \cdot \dot{y} \cdot h}{x_{M} \cdot y-y_{M} \cdot x}
\end{array}\right.
\end{aligned}
$$




\section{Geometric Method, Determining Angular Velocities and Accelerations}

Once determined velocities and accelerations $\mathrm{O}_{3}$ point, we can move on to determining the absolute angular velocities and angular accelerations of the system. It starts from the system (75), which is derived a function of time and get the system (87):

$$
\left\{\begin{array}{l}
\dot{x}=-d_{2} \cdot \sin \phi_{20} \cdot \dot{\phi}_{20} \\
\dot{y}=d_{2} \cdot \cos \phi_{20} \cdot \dot{\phi}_{20}
\end{array}\right.
$$

For correct solution of the system (87) mounts the first relationship to the $\left(-\sin \varphi_{20}\right)$ and the second with $\left(\cos \varphi_{20}\right)$, then collect both relationships obtained (member by member) and the explanation of the $\dot{\phi}_{20}$, obtained searched expression (88):

$$
\omega_{2} \equiv \omega_{20} \equiv \dot{\phi}_{20}=\frac{\dot{y} \cdot \cos \phi_{20}-\dot{x} \cdot \sin \phi_{20}}{d_{2}}
$$

The velocities system (87) is again derived the time and to obtain the absolute angular accelerations system (89):

$$
\left\{\begin{array}{l}
\ddot{x}=-d_{2} \cdot \cos \phi_{20} \cdot \dot{\phi}_{20}^{2}-d_{2} \cdot \sin \phi_{20} \cdot \ddot{\phi}_{20} \\
\ddot{y}=-d_{2} \cdot \sin \phi_{20} \cdot \dot{\phi}_{20}^{2}+d_{2} \cdot \cos \phi_{20} \cdot \ddot{\phi}_{20}
\end{array}\right.
$$

For correct solution of the system (89) amplify the first its relationship to the $\left(-\sin \varphi_{20}\right)$ and the second with $\left(\cos \varphi_{20}\right)$ (see equations from system 90), then collect both relationships obtained (member by member) and the explanation of the $\dot{\phi}_{20}$, obtained searched expression (91):

$$
\begin{aligned}
& \left\{\begin{array}{l}
\ddot{x}=-d_{2} \cdot \cos \phi_{20} \cdot \dot{\phi}_{20}^{2}-d_{2} \cdot \sin \phi_{20} \cdot \ddot{\phi}_{20} \mid \cdot\left(-\sin \phi_{20}\right) \\
\ddot{y}=-d_{2} \cdot \sin \phi_{20} \cdot \dot{\phi}_{20}^{2}+d_{2} \cdot \cos \phi_{20} \cdot \ddot{\phi}_{20} \mid \cdot\left(\cos \phi_{20}\right)
\end{array}\right. \\
& \varepsilon_{2} \equiv \varepsilon_{20} \equiv \dot{\omega}_{20} \equiv \ddot{\phi}_{20}=\frac{\ddot{y} \cdot \cos \phi_{20}-\ddot{x} \cdot \sin \phi_{20}}{d_{2}}
\end{aligned}
$$

Remember the two relationships in the system (92):

$$
\left\{\begin{array}{l}
\omega_{2} \equiv \omega_{20} \equiv \dot{\phi}_{20}=\frac{\dot{y} \cdot \cos \phi_{20}-\dot{x} \cdot \sin \phi_{20}}{d_{2}} \\
\varepsilon_{2} \equiv \varepsilon_{20} \equiv \dot{\omega}_{20} \equiv \ddot{\phi}_{20}=\frac{\ddot{y} \cdot \cos \phi_{20}-\ddot{x} \cdot \sin \phi_{20}}{d_{2}}
\end{array}\right.
$$

Still express Equation 93, (Fig. 3):

$$
\left\{\begin{array}{l}
x_{M}-x=d_{3} \cdot \cos \phi_{30} \\
y_{M}-y=d_{3} \cdot \sin \phi_{30}
\end{array}\right.
$$

The relationships of the system (93) further are derived with the time and obtained the velocities equations, system (94):

$\left\{\begin{array}{l}\dot{x}_{M}-\dot{x}=-d_{3} \cdot \sin \phi_{30} \cdot \dot{\phi}_{30} \\ \dot{y}_{M}-\dot{y}=d_{3} \cdot \cos \phi_{30} \cdot \dot{\phi}_{30}\end{array}\right.$

For correct solution of the system (94) amplify the first its relationship to the $\left(-\sin \varphi_{30}\right)$ and the second with $\left(\cos \varphi_{30}\right)$, then collect both relationships obtained (member by member) and the explanation of the $\dot{\phi}_{30}$, obtained wanted expression (95):

$\omega_{3} \equiv \omega_{30} \equiv \dot{\phi}_{30}=\frac{\left(\dot{y}_{M}-\dot{y}\right) \cdot \cos \phi_{30}-\left(\dot{x}_{M}-\dot{x}\right) \cdot \sin \phi_{30}}{d_{3}}$

It derives the then with the time, the velocities system (94) and the absolute angular accelerations Obtain system (96):

$\left\{\begin{array}{l}\ddot{x}_{M}-\ddot{x}=-d_{3} \cdot \cos \phi_{30} \cdot \dot{\phi}_{30}^{2}-d_{3} \cdot \sin \phi_{30} \cdot \ddot{\phi}_{30} \\ \ddot{y}_{M}-\ddot{y}=-d_{3} \cdot \sin \phi_{30} \cdot \dot{\phi}_{30}^{2}+d_{3} \cdot \cos \phi_{30} \cdot \ddot{\phi}_{30}\end{array}\right.$

For correct solution of the system (96) amplify the first its relationship to the $\left(-\sin \varphi_{30}\right)$ and the second with $\left(\cos \varphi_{30}\right)$, then collect both relationships obtained (member by member) and by the explanation of the $\ddot{\phi}_{30}$, obtained wanted expression (97):

$\varepsilon_{3} \equiv \varepsilon_{30} \equiv \dot{\omega}_{30} \equiv \ddot{\phi}_{30}=\frac{\left(\ddot{y}_{M}-\ddot{y}\right) \cdot \cos \phi_{30}-\left(\ddot{x}_{M}-\ddot{x}\right) \cdot \sin \phi_{30}}{d_{3}}$

Keep in the system (98) found the two solutions and in system (99) centralize all four of them:

$\left\{\begin{array}{l}\omega_{3} \equiv \omega_{30} \equiv \dot{\phi}_{30}=\frac{\left(\dot{y}_{M}-\dot{y}\right) \cdot \cos \phi_{30}-\left(\dot{x}_{M}-\dot{x}\right) \cdot \sin \phi_{30}}{d_{3}} \\ \varepsilon_{3} \equiv \varepsilon_{30} \equiv \dot{\omega}_{30} \equiv \ddot{\phi}_{30}=\frac{\left(\ddot{y}_{M}-\ddot{y}\right) \cdot \cos \phi_{30}-\left(\ddot{x}_{M}-\ddot{x}\right) \cdot \sin \phi_{30}}{d_{3}}\end{array}\right.$

$\left\{\begin{array}{l}\omega_{2} \equiv \omega_{20} \equiv \dot{\phi}_{20}=\frac{\dot{y} \cdot \cos \phi_{20}-\dot{x} \cdot \sin \phi_{20}}{d_{2}} \\ \omega_{3} \equiv \omega_{30} \equiv \dot{\phi}_{30}=\frac{\left(\dot{y}_{M}-\dot{y}\right) \cdot \cos \phi_{30}-\left(\dot{x}_{M}-\dot{x}\right) \cdot \sin \phi_{30}}{d_{3}} \\ \varepsilon_{2} \equiv \varepsilon_{20} \equiv \dot{\omega}_{20} \equiv \ddot{\phi}_{20}=\frac{\ddot{y} \cdot \cos \phi_{20}-\ddot{x} \cdot \sin \phi_{20}}{d_{2}} \\ \varepsilon_{3} \equiv \varepsilon_{30} \equiv \dot{\omega}_{30} \equiv \ddot{\phi}_{30}=\frac{\left(\ddot{y}_{M}-\ddot{y}\right) \cdot \cos \phi_{30}-\left(\ddot{x}_{M}-\ddot{x}\right) \cdot \sin \phi_{30}}{d_{3}}\end{array}\right.$ 


\section{Conclusion}

Today, anthropomorphic structures are used more and more in almost all the fields of industrial. Robotic structures have emerged from the need for automation and robotics of the industrial processes. The first industrial robots were called upon by the heavy industry and in particular by the automobiles industry. The automotive industry not only has requested the appearance of the industrial robots but even their subsequent development.

The most used were and have remained, the robots anthropomorphic, because they are more easily designed, built, maintained, are easily to handle, more dynamics, robust, economic and in general they have a broadly working area. The structures of the solid anthropomorphic robots are made up of elements and the couples of rotation, to which can add on an occasional basis and one or more couplers with translational moving. The couplers of rotation have been proven their effectiveness by moving them easier, more dynamic, step by step and especially being the most reliable. In general the couplers of rotation are moving more easily and more continuous, are actuated better and easier, control is less expensive and more reliable and programming the movements of rotation is also much simpler and more efficient.

This paper presents and treats (in an original way) the specific elements of the structures of robotic solid mobile anthropomorphic type. Are "placed on the wallpaper", the geometry, kinematics and dynamics of the anthropomorphic robotic solid systems, in an original vision of the authors. Last part presents the inverse kinematics of anthropomorphic systems, with mechanical elements and points: Geometry, cinematic, positions, displacements, velocities and accelerations, by two methods (as the most representatives): First one the method trigonometric and second one the geometric method.

\section{Acknowledgement}

This text was acknowledged and appreciated by Assoc. Pro. Taher M. Abu-Lebdeh, North Carolina A and T State Univesity, United States, Samuel P. Kozaitis, Professor and Department Head at Electrical and Computer Engineering, Florida Institute of Technology, United States, whom we thank and in this way.

\section{Funding Information}

Research contract: Contract number 36-5-4D/1986 from 24IV1985, beneficiary CNST RO (Romanian National Center for Science and Technology) Improving dynamic mechanisms.

Contract research integration. 19-91-3 from 29.03.1991; Beneficiary: MIS; TOPIC: Research on designing mechanisms with bars, cams and gears, with application in industrial robots.

Contract research. GR 69/10.05.2007: NURC in 2762; theme 8: Dynamic analysis of mechanisms and manipulators with bars and gears.

Labor contract, no. 35/22.01.2013, the UPB, "Stand for reading performance parameters of kinematics and dynamic mechanisms, using inductive and incremental encoders, to a Mitsubishi Mechatronic System" "PN-IIIN-CI-2012-1-0389".

All these matters are copyrighted! Copyrights: $394-$ qodGnhhtej, from 17-02-2010 13:42:18; 463vpstuCGsiy, from 20-03-2010 12:45:30; 631sqfsgqvutm, from 24-05-2010 16:15:22; 933CrDztEfqow, from 07-01-2011 13:37:52.

\section{Author's Contributions}

All the authors contributed equally to prepare, develop and carry out this manuscript.

\section{Ethics}

This article is original. Authors declare that are not ethical issues that may arise after the publication of this manuscript.

\section{References}

Angeles, J., 1989. An algorithm for the inverse dynamics of n-axis general manipulators using Kane's equations. Comput. Math. Applic., 17: 1545-1561. DOI: 10.1016/0898-1221(89)90054-0

Atkenson, C., H.A. Chae and J. Hollerbach, 1986. Estimation of Inertial Parameters of Manipulator Load and Links. 1st Edn., MIT Press, Cambridge, Massachuesetts.

Avallone, E.A. and T. Baumeister, 1996. Marks' Standard Handbook for Mechanical Engineers. 10th Edn., McGraw-Hill, New York, ISBN-10: 0070049971, pp: 1741.

Aversa, R., D. Parcesepe, R.V. Petrescu, G. Chen and F.I.T. Petrescu et al., 2016a. Glassy amorphous metal injection molded induced morphological defects. Am. J. Applied Sci., 13: 1476-1482. DOI: 10.3844/ajassp.2016.1476.1482

Aversa, R., F.I.T. Petrescu, R.V. Petrescu and A. Apicella, 2016b. Biomimetic finite element analysis bone modeling for customized hybrid biological prostheses development. Am. J. Applied Sci., 13: 1060-1067. DOI: 10.3844/ajassp.2016.1060.1067

Aversa, R., R.V. Petrescu, F.I.T. Petrescu and A. Apicella, 2016c. Smart-factory: Optimization and process control of composite centrifuged pipes. Am. J. Applied Sci., 13: 1330-1341.

DOI: 10.3844/ajassp.2016.1330.1341 
Aversa, R., F. Tamburrino, R.V. Petrescu, F.I.T. Petrescu and M. Artur et al., 2016d. Biomechanically inspired shape memory effect machines driven by Muscle like acting NiTi alloys. Am. J. Applied Sci., 13: 1264-1271.

DOI: 10.3844 ajassp.2016.1264.1271

Aversa, R., F.I.T. Petrescu, R.V. Petrescu and A. Apicella, 2016e. Biofidel FEA modeling of customized hybrid biological hip joint prostheses, Part I: Biomechanical behavior of implanted femur. Am. J. Biochem. Biotechnol., 12: 270-276. DOI: 10.3844/ajbbsp.2016.270.276

Aversa, R., F.I.T. Petrescu, R.V. Petrescu and A. Apicella, 2016f. Biofidel FEA modeling of customized hybrid biological hip joint design Part II: Flexible stem trabecular prostheses. Am. J. Biochem. Biotechnol., 12: 277-285. DOI: 10.3844/ajbbsp.2016.277.285

Baili, M., 2003. Classification of 3R Ortogonal positioning manipulators. Technical report, University of Nantes.

Baron, L. and J. Angeles, 1998. The On-Line Direct Kinematics of Parallel Manipulators Under JointSensor Redundancy. In: Advances in Robot Kinematics: Analysis and Control, Lenarčič, J. and M.L. Husty (Eds.), Springer, pp: 127-136.

Borrel, P. and A. Liegeois, 1986. A study of multiple manipulator inverse kinematic solutions with applications to trajectory planning and workspace determination. Proceedings of the IEEE International Conference on Robotics and Automation, Apr. 7-10, IEEE Xplore Press, pp: 1180-1185. DOI: 10.1109/ROBOT.1986.1087554

Burdick, J.W., 1988. Kinematic analysis and design of redundant manipulators. PhD Dissertation, Stanford.

Ceccarelli, M., 1996. A formulation for the workspace boundary of general n-revolute manipulators. Mechan. Mach. Theory, 31: 637-646. DOI: 10.1016/0094-114X(95)00096-H

Choi, J.K., O. Mori and T. Omata, 2004. Dynamic and stable reconfiguration of self-reconfigurable planar parallel robots. Adv. Robot., 18: 565-582. DOI: $10.1163 / 1568553041257440$

Denavit, J., 1964. Kinematic Syntesis of Linkage. 1st Edn., McGraw-Hill, Hartenberg R.SN.Y.

Di Gregorio, R. and V. Parenti-Castelli, 2002. Dynamic Performance Indices for 3-DOF Parallel Manipulators. In: Advances in Robot Kinematics, Lenarcic, J. and F. Thomas (Eds.), Kluver Academic Publisher, pp: 11-20.

Goldsmith, P.B., 2002. Kinematics and stiffness of a symmetrical 3-UPU translational parallel manipulator. Proceedings of the IEEE International Conference on Robotics and Automation, May 11-15, IEEE Xplore Press, Washington DC, pp: 4102-4107. DOI: 10.1109/ROBOT.2002.1014387
Grotjahn, M., B. Heimann and H. Abdellatif, 2004. Identification of friction and rigid-body dynamics of parallel kinematic structures for model-based control. Multibody Syst. Dynam., 11: 273-294. DOI: 10.1023/B:MUBO.0000029426.05860.c2

Guegan, S. and W. Khalil, 2002. Dynamic Modeling of the Orthoglide. In: Advances in Robot Kinematics, Lenarcic, J. and F. Thomas (Eds.), Kluver Academic Publisher, pp: 287-396.

Kim, H.S. and L.W. Tsai, 2002. Kinematic synthesis of spatial 3-RPS parallel manipulators. Proceedings of the Design Engineering Technical Conferences and Computers and Information in Engineering Conference, Sept. 29-Oct. 2, Canada, pp: 873-880. DOI: 10.1115/DETC2002/MECH-34302

Lee, W.H. and A.C. Sanderson, 2001. Dynamic analysis and distributed control of the tetrarobot modular reconfigurable robotic system. Autonomous Syst., 10: 67-82.

Liu, X.J. and J. Kim, 2002. A new three-degree-offreedom parallel manipulator. Proceedings of the IEEE International Conference on Robotics and Automation, May 11-15, IEEE Xplore Press, pp: 1155-1160. DOI: 10.1109/ROBOT.2002.1014699

Lorell, K., J.N. Aubrun, R.R. Clappier, B. Shelef and G. Shelef, 2003. Design and preliminary test of precision segment positioning actuator for the California Extremely Large Telescope. Proc. SPIE, 4840: 471-484. DOI: 10.1117/12.459974

Merlet, J.P., 2000. Parallel Robots. 2nd Edn., Kluver Academic Publisher, Dordrecht, ISBN-10: 0792363086, pp: 335.

Miller, K., 2004. Optimal design and modeling of spatial parallel manipulators. Int. J. Robot. Res., 23: 127-140. DOI: 10.1177/0278364904041322

Petrescu, F.I., B. Grecu, A. Comănescu and R.V. Petrescu, 2009. Some mechanical design elements. Proceedings of International Conference Computational Mechanics and Virtual Engineering, (MVC' 09), Braşov, Romania, pp: 520-525.

Tsai, L.W., 2000. Solving the inverse dynamics of a Stewart-Gough manipulator by the principle of virtual work. ASME J. Mechan. Design, 122: 3-9. DOI: $10.1115 / 1.533540$ 